\title{
Ilíada VI (407-439): la auto-configuración femenina de Andrómaca
}

Iliad VI (407-439): the Andromache's female self-configuration

\author{
Deidamia Sofia Zamperetti Martin* \\ Universidad Nacional de La Plata, Argentina \\ zamperettimartin@fahce.unlp.edu.ar
}

\section{ReSUMEN:}

En el canto VI de Ilíada, Héctor y Andrómaca representan el lado íntimo de la guerra. Abordaremos aquí la auto-configuración del personaje de Andrómaca como mujer atendiendo a los distintos sentidos que asumen sus propias palabras. Nos proponemos estudiar, a partir de un análisis filológico-literario, el discurso del personaje de Andrómaca en el canto VI de Ilíada (407-439) teniendo en cuenta diversos recursos de análisis sintáctico, semántico y pragmático. El objetivo de esta investigación es detectar e indagar las estructuras predicativas y sus constituyentes -obligatorios o no- para dilucidar la funcionalidad de las mismas en relación con las distintas partes del discurso.

Palabras Clave: Homero, Ilíada, Andrómaca.

\section{Abstract:}

In the book VI of Iliad, Hector and Andromache represent the intimate side of war. Here we will address the self-configuration Andromache's character as a woman focusing on to the various senses that their own words assume. We intend to study, from a philological-literary analysis, the speech of Andromache in the Iliad, book VI (407-439), considering various resources of syntactic, semantic and pragmatic analysis. The aim of this research is to detect and analyse the predicative structures and their constituents (required or not) to elucidate their functionality in relation to the different parts of speech.

KEYWORDS: Homer, Iliad, Andromache.

Al promediar la segunda parte del canto VI de Ilíada, asistimos a una serie de encuentros entre Héctor, el héroe troyano por excelencia, y diversos integrantes de su familia. La tercera de las entrevistas que el héroe lleva a cabo dentro de la ciudadela de Troya se trata del encuentro con su esposa, Andrómaca, que más allá de acercarnos a una visión de la guerra desde la perspectiva de los defensores de la ciudad, nos expone las problemáticas propias del ámbito marital en el contexto bélico.

En una instancia anterior, en el marco de nuestros estudios acerca de Ilíada, hemos propuesto una interpretación de este pasaje, a partir del análisis filológico-literario del encuentro entre los esposos (407-493), centrando nuestro interés en la configuración heroica del personaje de Héctor. ${ }^{1}$ En esta oportunidad, realizaremos un nuevo reconocimiento del texto griego para analizar específicamente el discurso de Andrómaca (407-439), con especial atención en las estructuras predicativas y sus constituyentes -obligatorios o no- y cómo funcionan en relación con la organización discursiva. Para ello utilizaremos algunos de los diversos recursos que nos ofrecen los tres niveles de análisis lingüístico: el sintáctico, el semántico y el pragmático. ${ }^{2}$

El encuentro entre los esposos, que tradicionalmente suele denominarse "coloquio de despedida", funciona desde el punto de vista argumental, en la macro-estructura del poema, principalmente, como una especie de entrevista pre-funeral. Extrapolando el discurso de Andrómaca, es posible plantear un análisis desde una perspectiva que contemple la construcción de un sujeto femenino en el contexto bélico-heroico a partir de su auto-relato (o el relato sobre sí mismo) en primera persona singular (género femenino), ${ }^{3}$ en que predominan la auto-referencia y la constante interlocución con la segunda persona del singular (género masculino). Este hecho fortalece la performance del íntimo vínculo que une a los esposos y pone en escena un ámbito de la esfera privada, poco usual en el contexto de la épica heroica. 
En primer lugar, es preciso señalar que el discurso de Andrómaca se adapta a la estructura retórica anular presentada en esquema tripartito que plantea Bakker, ${ }^{4}$ quien sostiene que los discursos se componen con una estructura típica. Constan de una primera parte denominada A (framing), que enmarca el discurso en un tema en particular adelantando el desarrollo del mismo; una segunda parte, B (link), constituida por una narración similar a la diégesis retórica que hace avanzar el discurso hacia su objetivo y, una tercera parte, $\mathrm{C}$ (goal), que retoma lo pronunciado en $\mathrm{A}$, dándole un nuevo sentido al que se ha arribado mediante la parte narrativa.

Asimismo, si confrontamos el discurso de Andrómaca con la subsiguiente respuesta de Héctor -que abarca los versos 441 a 465, 476 a 481 y 486 a 493- resulta clara la aplicabilidad del esquema que, de modo comparativo, constituye la propuesta de Lohmann, ${ }^{5}$ donde A (407-411) se corresponde con A' (454-465), B (411-430) con B' (447-454) y C (431-439) con C' (441-446), en consonancia con la habitual organización retórica de los discursos en Homero. ${ }^{6}$

Ahora bien, a partir del estudio del discurso desde el punto de vista de las funciones comunicativas, ${ }^{7}$ podemos estructurar la intervención de Andrómaca planteando tres momentos: una primera instancia donde predomina la función comunicativa asertiva/declarativa (407-430), ${ }^{8}$ un segundo y breve momento netamente impresivo (431-434) y, ${ }^{9}$ por último, una tercera instancia asertiva/declarativa (435-439) que funciona como síntesis y clausura del discurso.

\section{UNO: VERSOS 407-430}

Luego de emplear un análisis de tipo oracional en el fragmento seleccionado, es decir, después de registrar cada constituyente de la oración -cada predicado ${ }^{10}$ con sus argumentos ${ }^{11}$ y la presencia no solo de adjuntos, ${ }^{12}$ sino también de disjuntos- ${ }^{13}$ podemos sostener que la primera sección del discurso se organiza en torno a tres disjuntos en posición inicial del verso, ${ }^{14}$ estructurando esta primera parte del discurso en tres sub-secciones: la primera, referida al futuro de Héctor (407-413); la segunda, concerniente al pasado de Andrómaca en vinculación con Aquiles (414-428) y, la tercera, en donde se retoma la figura de Héctor pero en el tiempo presente de la enunciación (429-430). Es llamativa la escasa presencia de disjuntos, tan solo tres en este discurso que, por su alto grado emotivo, podría haberse encontrado repleto de este tipo de elementos.

El término inicial con que Andrómaca se dirige a Héctor es claramente un disjunto, en este caso referido a uno de los protagonistas de la comunicación -el destinatario del discurso de Andrómaca- y es la invocación $\delta \alpha \mu o_{v i \varepsilon}(407) .{ }^{15}$ Mediante esta realización léxica, Andrómaca apela a su interlocutor caracterizando al personaje de Héctor de una manera peculiar al referirse a su mala fortuna y, por extensión, a las circunstancias desdichadas en que se enmarca la emisión de su discurso:

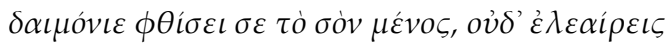

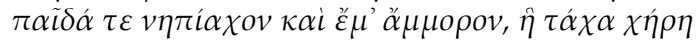

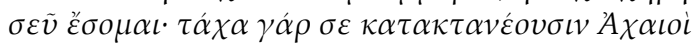

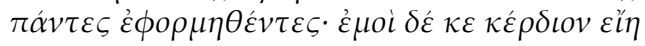

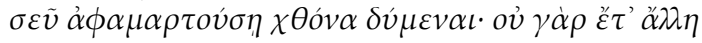

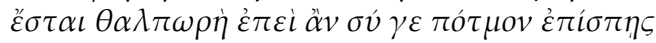

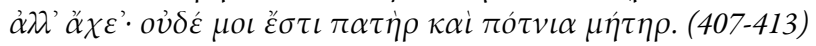

Desgraciado, tu vigor te destruirá y no compadeces al pequeño hijo ni a mi, desventurada, que rápidamente seré tu viuda. Pues pronto los aqueos te matarán, lanzándose todos a la vez. Y sería más ventajoso para mí, si te pierdo, sumergirme en la tierra. Pues ya no habrá ningún otro alivio cuando tú en efecto, eventualmente, llegues a tu muerte, sino penas. Pues no tengo padre ni venerable madre. 


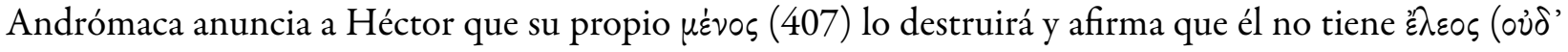
$\dot{\varepsilon} \lambda \varepsilon \alpha i p \varepsilon ı \varsigma, 407){ }^{17}$ ni por su hijo ni por ella misma que se convertirá en $\chi \dot{\eta} p \eta$ (408) a causa de que los aqueos matarán a su esposo y la tomarán prisionera. Es notable que, en consonancia con la intención comunicativa que señalamos para esta primera parte del discurso, prevalece el uso de formas verbales de tiempo futuro del indicativo y presente del indicativo. ${ }^{18} \mathrm{El}$ grado de inmediatez de los trágicos hechos que se avecinan queda retratado mediante la repetición del adjunto $\tau \dot{\alpha} \chi \alpha \alpha$ en los versos 408 y 409 , que pone de relieve la inminencia de la muerte del ser amado. Además, entre los versos 408 y 411 hallamos dos predicativos objetivos ( $\nu \eta \pi i \alpha \chi \circ v$,

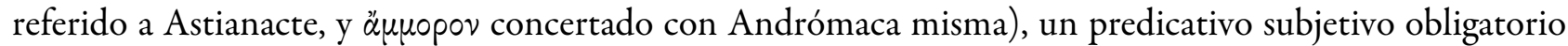

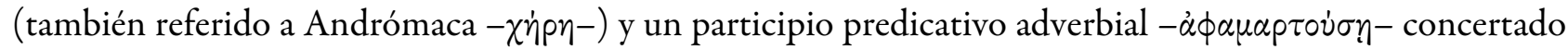
con el objeto indirecto éroi (410) que colaboran en la descripción y presentación del estado de cosas.

El fragmento comprendido entre los versos 414 y 428 se inicia con el disjunto ク̈ंo que expresa un valor de verdad comprometido en el enunciado por parte del emisor. Andrómaca se manifiesta carente de vínculos familiares porque Aquiles los ha aniquilado a todos y resume que la carencia de estos vínculos ha sido suplantada por la figura de Héctor, ${ }^{19}$ quien encarna su familia por completo, oficiando de padre, madre, hermano, además de ser su esposo:

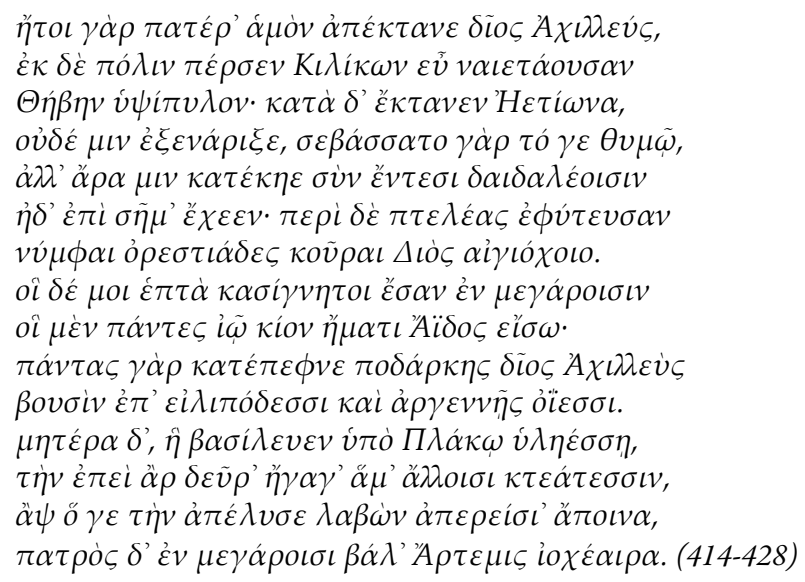

Pues, en verdad, el divino Aquiles mató a mi padre y destruyó totalmente la ciudad de los Cilicios, bella de habitar, Tebas, la de las altas puertas. Y asesinó a Etión y no lo despojó pues temió algo en su ánimo, sino que lo quemó con sus labradas armas y erigió un túmulo sobre el cuerpo. Alrededor, las ninfas que habitan los montes, hijas de Zeus que lleva la égida, plantaron olivos. Estos que eran mis siete hermanos en los palacios, todos, marcharon al Hades el mismo día a causa de la flecha. Pues a todos mató el divino Aquiles, el de los pies ligeros, entre bueyes de pasos rodantes y blancas ovejas. A mi madre, que reinaba en la selvosa Placo, a esta, después de que la condujo hasta aqui junto con otras propiedades, este efectivamente la liberó tomando un inmenso rescate y Artemis, tiradora de flechas, la hirió en los palacios de su padre.

El razonamiento implícito en este fragmento permite sostener que, ya que Aquiles ha sido el asesino de todos sus seres amados en el pasado, se preanuncia -de alguna manera- que también será el asesino de Héctor, es decir, como Aquiles mató a la familia de Andrómaca y ahora Héctor representa a su familia, entonces Aquiles también aniquilará a Héctor.

Es preciso señalar la abundancia de circunstanciales de lugar que especifican detalles geográficos o espaciales relativos a la muerte de sus seres queridos y colaboran en la descripción de los eventos del pasado, ${ }^{20}$ narrados a través de una reiterada presencia de predicados en tiempo aoristo del modo indicativo. ${ }^{21}$ Por otra parte, es llamativa la repetición de aposiciones: en primer lugar, en el verso 416, la aposición referida a la ciudad de 
los Cilicios, $\Theta \dot{\eta} \beta \eta v \dot{\gamma} \psi i \pi v \lambda \circ \nu$ (“Tebas, la de las altas puertas”); en segundo lugar, en el verso 420, la aposición

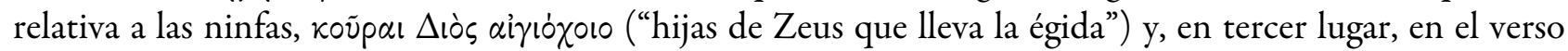

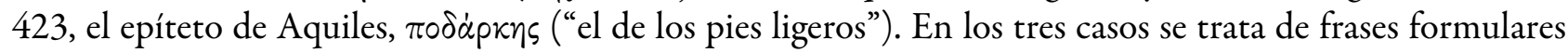
de tipo convencional en la épica, mientras que, en el verso 425 hallamos el sustantivo $\mu \eta \tau \dot{p} \rho \alpha$ ("madre")

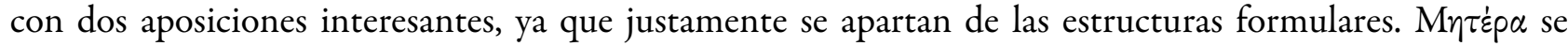
recupera a través del pronombre anafórico $\tau \grave{\eta} \nu$ (426) funcionando sintácticamente como aposición y repetido enfáticamente en el verso 427 con función de objeto directo o argumento del predicado $\dot{\alpha} \pi \dot{\varepsilon} \lambda \nu \sigma \varepsilon$. En este sentido, los pronombres anafóricos que recuperan $\mu \eta \tau \dot{\varepsilon} \rho \alpha$ como referente rompen el orden lógico de la estructura sintáctica, poniendo en evidencia el $\pi \dot{a} \theta \circ \varsigma$ de Andrómaca al referirse a la muerte de su madre en la antesala de la muerte de su esposo.

Los dos versos que finalizan esta primera sección del discurso de Andrómaca se encuentran encabezados por el disjunto Ekтop (429), se trata de una invocación que refuerza la apelación de Andrómaca a su esposo, ${ }^{22}$ lo exhorta en consonancia con el acto de habla indirecto que transmite su discurso, ${ }^{23}$ en este caso, intentar persuadir al interlocutor para que actúe de la manera que se le propone:

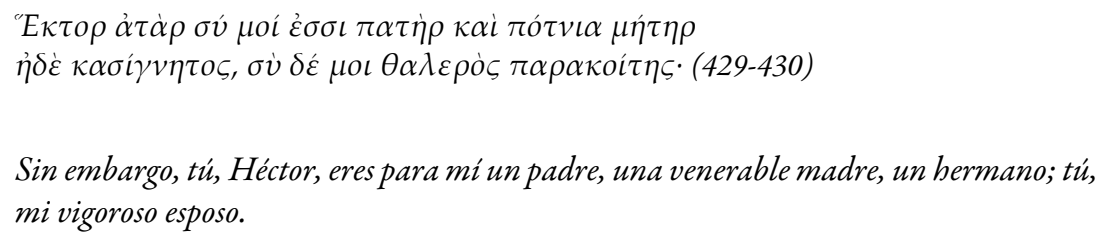

El caso vocativo posee cierta independencia sintáctica, no está regido directamente por el predicado de la frase, es decir, que no rellena ninguna casilla argumental del marco predicativo de un verbo, ni es un adjunto, pero se integra a la frase como un disjunto, es por eso que debe ser considerado en el plano de la actuación cumpliendo así un papel similar al del imperativo en el ámbito verbal. ${ }^{24}$ El uso del vocativo se encuentra estrechamente ligado a la fuerza ilocutiva que posee en el contexto en que aparece, por lo tanto, el nivel de análisis más apropiado es el del discurso, en que hay un hablante y un oyente. ${ }^{25}$

Es interesante, además, señalar la reiteración de los pronombres personales de segunda persona del singular en caso nominativo $\left(\sigma \dot{)}\right.$ y de primera persona del singular en dativo ( $\left.\mu o^{\prime}\right)$ en los versos 429 y 430 , exhibiendo una estructura balanceada en que semánticamente quedan determinados $\sigma u ́$ como sujeto y $\mu$ oí como beneficiario en cuanto a sus funciones semánticas. ${ }^{26}$ En lenguas como el español, el griego o el latín, en que las distintas personas están expresadas por las desinencias verbales, si aparece un pronombre personal en caso nominativo resulta redundante y añade cierto énfasis, especialmente, cuando se contraponen distintas personas. $^{27}$

Observamos en el discurso de Andrómaca una especie de juego de alternancias entre los pronombres personales de primera y segunda persona, donde hallamos siete registros de formas del pronombre $\dot{\varepsilon} \gamma \omega^{28}$ y la misma cantidad de apariciones de $\sigma v^{2}{ }^{29}$ Resulta altamente significativo que el pronombre de primera persona, en cinco de los registros, esté funcionando en estructuras del verbo eíú acompañado de un dativo, como ocurre en el caso de los versos $429-430$ referido anteriormente. ${ }^{30}$

Con respecto a la disposición de los constituyentes en las oraciones, es decir, el orden de palabras, podemos señalar que, en este juego de alternancias, los pronombres personales se ubican, generalmente, a mitad del hexámetro dactílico, salvo dos excepciones en la sección inicial del discurso donde el pronombre de la segunda persona del singular en genitivo se posiciona al inicio del verso generando mayor énfasis en la misma. ${ }^{31}$ 


\section{Dos: VeRsos 431-434}

En la segunda sección de la estructura compositiva que planteamos para el discurso de Andrómaca, comprendida entre los versos 431 y 434, el interés del hablante por persuadir a su interlocutor aparece más claramente expuesto con una serie de verbos imperativos: $\dot{\varepsilon} \lambda \dot{\varepsilon} \alpha \iota p \varepsilon$ y $\mu$ i $\mu \nu \varepsilon$ (ambos en presente de imperativo, verso 431), $\theta \dot{\eta} \eta s$ (si bien es un aoristo subjuntivo, el modo sintáctico es prohibitivo, verso 432) y $\sigma \tau \tilde{\eta} \sigma o v$ (aoristo imperativo, verso 433)..$^{32}$

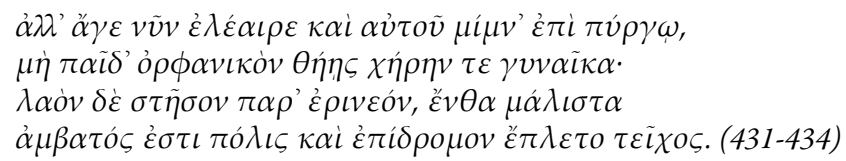

Pero vamos, compadécete ahora de ti mismo y permanece sobre la torre, no hagas huérfano a un hijo y viuda a una mujer. Coloca el ejército junto a la higuera, alli donde la ciudad es más fácil de escalar y el muro se eleva accesible.

Pues, luego de llegar, los mejores lo intentaron tres veces, junto a los dos Áyax y el glorioso Idomeneo y junto a los Atridas y el fuerte hijo de Tideo. Ya sea que lo anunció alguien que conoce bien los oráculos, ya sea que ahora el ánimo de estos los anime e impulse.

Cuando Andrómaca utiliza el imperativo é $\lambda \dot{\varepsilon} \alpha \iota p \varepsilon(431),{ }^{33}$ apela a que Héctor se compadezca de ella y de su hijo, reclamando, mediante $\mu i \mu \nu \varepsilon$ (431), que su esposo permanezca dentro de la ciudadela para que no la convierta en viuda y a su hijo en huérfano ( $\theta \dot{\eta} n$ s, 433). Es atractiva la presencia del adverbio vข̃v en el verso 431, ya que permite una doble interpretación: por un lado, como un circunstancial de tiempo (adjunto vinculado al predicado) que simplemente restringiría en el tiempo la acción de "compadecerse" y, por otro lado, como un disjunto que intensifica la urgencia del hecho de que Héctor se compadezca, referido a la circunstancia de la emisión. Nos inclinamos hacia esta segunda posibilidad, especialmente, por la presencia

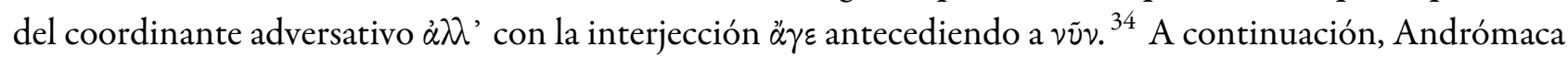
proporciona a Héctor una indicación de estrategia bélica mediante el predicado $\sigma \tau \tilde{\eta} \sigma o v: \lambda \alpha o ̀ \nu ~ \delta \dot{\varepsilon} \sigma \tau \tilde{\eta} \sigma o \nu \pi \alpha \rho^{\prime}$

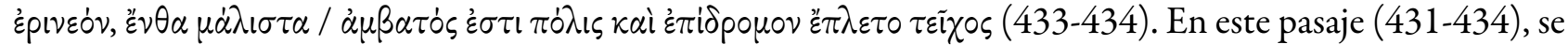
evidencia que las percepciones acerca de la guerra no son las mismas para los hombres que para las mujeres. Las consecuencias que pueden padecerse ante una resolución bélica adversa son diferentes: los hombres luchan por la defensa de la ciudad y mueren en el combate encarnizado; mientras que las mujeres dedican todo su esfuerzo al sustento de la casa y la familia, temiendo que sus hijos y esposos mueran y que ellas puedan llegar a convertirse en esclavas del enemigo. Héctor y Andrómaca, en la íntima escena del canto VI, reproducen estas dos perspectivas de la guerra donde cada uno manifiesta las problemáticas de su propio género.

Entre los versos 431 y 434, cuando la función del discurso es netamente impresiva, predominan las predicaciones nucleares, Andrómaca resulta sintética y prioriza transmitir la información básica de la predicación.

\section{Tres: VERSOS 435-439}

Como anticipamos, el tercer momento del discurso de Andrómaca (435-439) contiene una síntesis y la clausura del mismo a través de la función comunicativa asertiva/declarativa: 


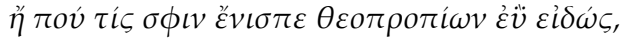

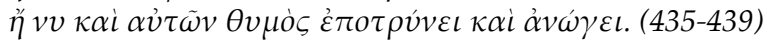

Pues, luego de llegar, los mejores lo intentaron tres veces, junto a los dos Ayax y el glorioso

Idomeneo y junto a los Atridas y el fuerte hijo de Tideo. Ya sea que lo anunció alguien

que conoce bien los oráculos, ya sea que ahora el ánimo de estos los anime e impulse.

Cuando Andrómaca articula nuevamente un discurso declarativo, reaparecen las predicaciones extendidas. En esta sección pueden diferenciarse dos partes: una vinculada al pasado rotundo, expresado con

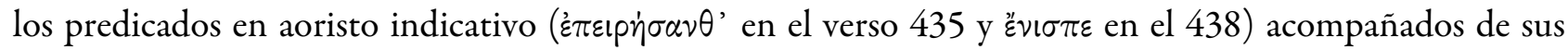
respectivos argumentos y de una numerosa cantidad de adjuntos referidos al enemigo. ${ }^{35}$ Otra, la segunda parte, caracterizada por un retorno al presente de la enunciación con la presencia de la partícula $v v$ en el verso

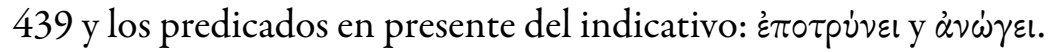

A modo de conclusión, sostenemos que mediante la intervención de Andrómaca, en el canto VI de Ilíada, asistimos a una de las escasas ocasiones en que una mujer desarrolla un discurso desde su óptica subjetiva femenina en el contexto de una narración bélica-heroica. A través de su relato, se erige un sujeto femenino con una historia personal en un poema épico en que, generalmente, la voz de la mujer se circunscribe a los actos de súplica y de lamento. El discurso de Andrómaca amplifica y contiene la historia de otras tantas mujeres anónimas en los poemas épicos.

En el discurso analizado predomina la auto-referencialidad sustentada, por ejemplo, en la repetición del pronombre personal de primera persona del singular que, junto a la reiterada presencia del pronombre de segunda persona del singular fortalece la representación del íntimo vínculo marital y pone en relieve una escena de atípica intimidad o domesticidad relativa al ámbito privado de la pareja troyana.

Un análisis integral del discurso desde el punto de vista sintáctico, semántico y pragmático enriquece sus posibilidades de interpretación al contemplar los diversos matices de expresión, de organización del contenido y de transmisión de información en los parlamentos de los personajes, en este caso, de un personaje femenino tan relevante como Andrómaca en la épica homérica. Las tres instancias de su discurso, con su circularidad asertiva la vuelven un ícono, símbolo o síntesis de muchas vidas femeninas; pero, al mismo tiempo, se cumple una construcción de su yo femenino que resulta único y peculiar.

\section{BIBLIOGRAFÍA}

Álvarez Huerta, O. (2009) “Pronombres”, en J. M. Baños Baños (coord.) Sintaxis del latín clásico, Madrid: 273-298.

Bakker, E. (1997) poetry in speech. Orality and Homeric discourse, Ithaca-London.

Baños Baños, J. M. (2009) “Dativo”, en J. M. Baños Baños (coord.) Sintaxis del latín clásico, Madrid: 185-209.

Baños Baños, J. M. y Cabrillana Leal, C. (2009) “Orden de palabras”, en J. M. Baños Baños (coord.) Sintaxis del latín clásico, Madrid: 679-707.

Cabrillana Leal, C. (2009) “Nominativo y Vocativo”, en J. M. Baños Baños (coord.) Sintaxis del latín clásico, Madrid: 111-130.

Graziosi, B. \& Haubold, J. (2010) Homer: Iliad. Book VI, Cambridge.

Kirk, G. S., Edwards, M. W., Janko, R., Hainsworth, V. \& Richardson, N. J. (1985-1993) The Iliad: A Commentary, Cambridge.

Leaf, W. (1900-1902) The Iliad. Volumes I and II, London.

Liddell, H. G. \& Scott, R. (1961) A Greek-English Lexicon, Oxford.

Lohmann, D. (1988) Die Andromache-Szenen der Ilias, Hildesheim-Zürich-New York.

Munro, D. B. \& Allen, T. W. (1920) Homer. Homeri Opera in five volumes, Oxford. 
Torrego Salcedo, M. E. y de la Villa Polo, J. (2009) “La oración: concepto. Estructura, constituyentes y niveles. Tipos”, en J. M. Baños Baños (coord.) Sintaxis del latín clásico, Madrid: 55-82.

Zamperetti martín, D. S. (2016) "Ilíada, VI: La configuración heroica de Héctor", en Actas del Séptimo Coloquio Internacional "[Una] nueva visión de la cultura griega antigua en el comienzo del tercer milenio: perspectivas y desafios", La Plata.

Zecchin de Fasano, G. C. (2002) “Temor y compasión en los poemas homéricos”, Synthesis Vol. 9: 109-128.

\section{Notas}

* Profesora y Licenciada en Letras por la Universidad Nacional de La Plata. Ayudante Diplomada del Área Griego de la Facultad de Humanidades y Ciencias de la Educación (UNLP). Secretaria e Investigadora del Centro de Estudios Helénicos del IdIHCS (UNLP-CONICET). Becaria CIN durante el período 2011-2012. Actualmente integra el proyecto de incentivos H861 "Del Treno al Epitafio: poética del lamento funeral en la literatura griega clásica. Inflexiones" (2018-2021). Entre sus publicaciones se encuentran Troya bifronte. Dicotomía y estética del relato de guerra

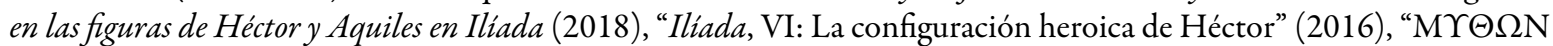
PHTSP: Fénix en Iliada IX, 434-605” (2015) y "Aproximaciones en torno a la figura de Aquiles en el Canto IX de Ilíada" (2013).

1 Cf. Zamperetti Martín (2015).

2 Cf. Torrego Salcedo y de la Villa Polo (2009: 63), donde se afirma que todos los constituyentes de una oración referidos a un predicado (argumentos, adjuntos y disjuntos) pueden analizarse desde el punto de vista sintáctico, semántico y pragmático. Sin embargo, no trabajaremos, por ejemplo, con las nociones de "tópico y foco", ya que en una estructura poética bastante rígida, como el hexámetro dactílico, el orden de palabras queda constreñido en muchas ocasiones por las necesidades métricas.

3 Si bien no existe ninguna variación o marca formal en el pronombre personal para la expresión del género, asignamos al referente la cualidad de "femenino" o "masculino" de acuerdo con la información que nos provee el contexto discursivo.

4 Cf. Bakker (1997: 116 y ss.) para un planteo general de la estructura de los discursos en Iliada.

5 Cf. Lohmann (1988: 34-35) para, específicamente, el discurso que nos ocupa y la respuesta al mismo.

6 Nótese que Lohmann solo contempla en su estructura la primera parte de la respuesta de Héctor (441-465), mientras que nosotros, además, incorporamos los versos subsiguientes (476-481 y 486-493). Al respecto cf. Zamperetti Martín (2015).

7 Cf. Torrego Salcedo y de la Villa Polo (2009: 75), donde se define la función comunicativa como la intención con que el hablante dirige el mensaje al destinatario.

8 Cf. Torrego Salcedo y de la Villa Polo (2009: 75): “(...) la intención del emisor en los enunciados asertivos es aumentar el conocimiento de su destinatario".

9 Cf. Torrego Salcedo y de la Villa Polo (2009: 76): "Mediante una ilocución impresiva, el hablante o emisor no pretende aumentar la información del destinatario, sino que trata de obtener una determinada actuación por su parte, es decir, pretende influir en su conducta".

10 Cf. Torrego Salcedo y de la Villa Polo (2009: 61): "El predicado es usualmente una forma verbal personal o un infinitivo, participio, etc. cuando la estructura predicativa es una oración independiente o una frase subordinada", y (2009: 62): "El predicado es el elemento central de la predicación y determina las características básicas de los constituyentes obligatorios que deben acompañarle".

11 Los argumentos son los constituyentes obligatorios de una oración. Cf. Torrego Salcedo y de la Villa Polo (2009: 64-66).

12 Los adjuntos, del mismo modo que los predicados con sus argumentos (predicación nuclear), pertenecen al nivel representativo pero se diferencian de los argumentos por su no obligatoriedad y son considerados como constituyentes de la predicación expandida, porque mantienen relación semántica con el predicado. Cf. Torrego Salcedo y de la Villa Polo (2009: 67-73).

13 Los disjuntos son considerados constituyentes del nivel interactivo, ya que hacen referencia a diversos aspectos propios de la comunicación, por ejemplo, a los participantes (hablante-destinatario), al valor de verdad del enunciado o a circunstancias relativas a su emisión. Cf. Torrego Salcedo y de la Villa Polo (2009: 73-75).

14 Nótese la importancia de la posición de los disjuntos como foco informativo. Cf. Baños Baños y Cabrillana Leal (2009: $680,685$ y 699$)$.

15 Citamos el texto griego de la edición oxoniense de Munro y Allen (1920). Asimismo, hemos consultado las ediciones de Kirk (1985-1993), Leaf (1900-2) y Graziosi y Haubold (2010).

16 La traducción de los textos griegos nos pertenece.

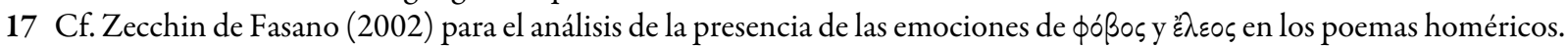




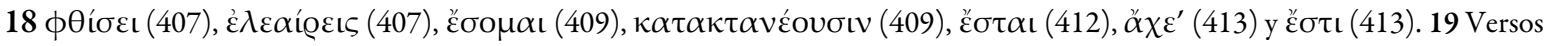
429-430.

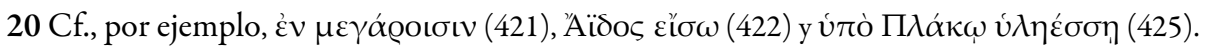

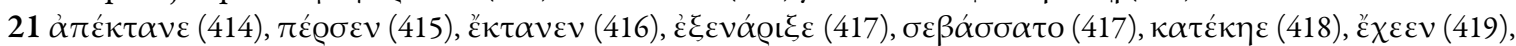

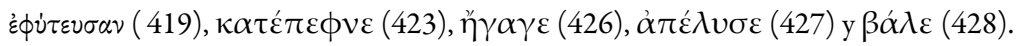

22 Acerca del uso del caso vocativo, cf. Cabrillana Leal (2009: 121-130).

23 Cf. Torrego Salcedo y de la Villa Polo (2009: 78-79).

24 Cf. Cabrillana Leal (2009: 121).

25 Cf. Cabrillana Leal (2009: 126).

26 Cf. Torrego Salcedo y de la Villa Polo (2009: 66-73).

27 Cf. Álvarez Huerta (2009: 285-287).

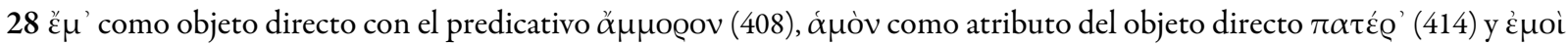

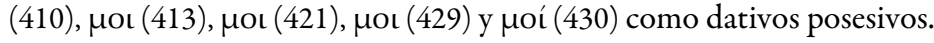

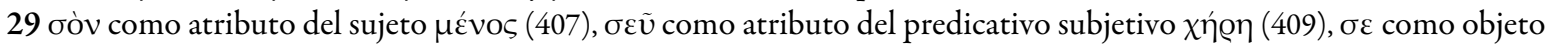

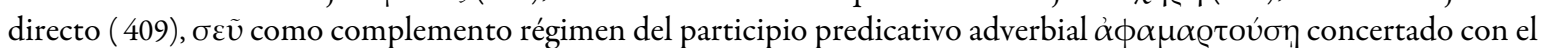
objeto i ndirecto éuoì (411) y oú (412, 429 y 430) como sujetos.

30 Para la problemática de determinar la función semántica del dativo en estructuras de sum más dativo cf. Baños Baños (2009: 197-198).

31 Versos 409 y 411 . Teniendo en cuenta el aspecto performativo de la épica, debemos considerar la posición de las palabras o el orden de los constituyentes en relación con el verso heroico y no ya en función de la organización oracional.

32 Es llamativo que, contrario a lo que señalan Baños Baños y Cabrillana Leal (2009: 696), los verbos de las oraciones impresivas en el discurso de Andrómaca no ocupan la posición inicial de frase (ni del verso), perdiendo, de esta forma, relevancia con un efecto suavizante de las órdenes.

33 Nótese cómo se recupera, mediante el predicado, la problemática que se plantea desde el primer verso del discurso (oủ ¿'

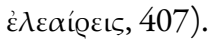

34 Para los diversos valores de võv, cf. la entrada léxica en Liddell y Scott (1961: 1185).

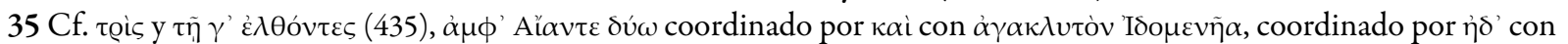

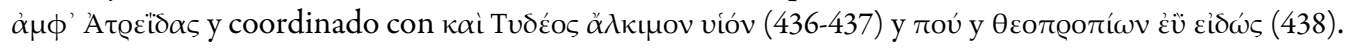

